\title{
Computational Agents to Model Knowledge - Theory, and Practice in Visual Surveillance
}

\author{
José Mira $^{1, \star}$, Ana E. Delgado ${ }^{1}$, Antonio Fernández-Caballero ${ }^{2}$, \\ José M. Gascueña ${ }^{2}$, and María T. López ${ }^{2}$ \\ 1 Departamento de Inteligencia Artificial, E.T.S.I. Informática, \\ Universidad Nacional de Educación a Distancia, 28040-Madrid, Spain \\ adelgado@dia.uned.es \\ 2 Universidad de Castilla-La Mancha, Departamento de Sistemas Informáticos \& \\ Instituto de Investigación en Informática de Albacete, 02071-Albacete, Spain \\ \{caballer, jmanuel,mlopez\}@dsi.uclm.es
}

\begin{abstract}
In this work the concept of computational agent is located within the methodological framework of levels and domains of description of a calculus in the context of different usual paradigms in Artificial Intelligence (symbolic, situated, connectionist, and hybrid). Emphasis in the computable aspects of agent theory is put, leaving open the possibility to the incorporation of other aspects that are still pure cognitive nomenclature without any computational counterpart of equivalent semantic richness. These ideas are currently being implemented on semiautomatic video-surveillance.
\end{abstract}

Keywords: Computational Agents, Knowledge Engineering, Artificial Intelligence, Visual surveillance.

\section{Introduction}

The concept of agent comes from the persistent attempt in Science and Engineering to modularize the knowledge necessary to specify a calculus, and of the later attempt to progressively increase the level of complexity and autonomy, making them re-usable. Agent theory takes from Artificial Intelligence (AI) the general intention to approach the functionality of biological systems. Thus, there are adaptive, intelligent, intentional agents, with learning capacity and equipped with a certain level of social organization that allows cooperation in the accomplishment of "group tasks". In this sense, the objectives of multi-agent systems (MAS) agree with distributed AI (DAI) 27. "Nature-inspired computation" 18] is also practically isomorphic to agent and MAS theory. This is true at the level of individual agents ("organisms") as well as at the level of social organizations in MAS (ants, bees, human societies, collective games, etc.). In this last case, to the functionalities demanded for the individual behavior, it is necessary to

\footnotetext{
* This article is dedicated to the memory of Professor José Mira, a great researcher, a wise man, a loving husband, and a close friend; but who sadly passed away. 
add the use of an interaction language among agents that allows to share goals and to coordinate the collective plans used to reach those goals. But, usually the specification and the modeling of the environment is forgotten. However, its richness, diversity and other characteristics are fundamental to understand the dynamics of the agent-system interaction.

In this work we approach the general concept of agent from a computational perspective. We consider that an agent starts being a conceptual model, later it is reduced to a formal model and finally to a physical machine with sensors, effectors and a control program. The agent concept is located within the methodological framework of description levels and domains of a calculus. Independently of the cognitive characteristics being assigned to an agent (intentions, purposes, beliefs, desires, emotions, or conscience), to our opinion an agent is a "computational agent", which performs a calculation in a physical machine having an interface (human or electromechanical) with an external environment.

\section{Computational Agents in Perspective}

Historically, Cybernetics introduced the concept of system, clearly analogous to the agent one. Physics and Engineering continue preferring to use the system concept, to which less cognitive characteristics are assigned. On the other hand, it is much more precise and operational, since its dynamics can be described in terms of differential equations - usual in Physics and Control Theory - and of combinatory and sequential logic - proper of computation and integrated in finite state automata (FSA) theory. In the end, when reformulating any definition in natural language of an agent in a computable form, it is also ended up using algorithms and automata. The initial cybernetical proposal consisting in studying the representation, calculation, communication and control systems, can now be rewritten in terms of agents to obtain a first general agent classification: representation, calculation, communication and control agents. Each one of these functions is characterized to have a clear objective (a goal), and to implicitly take the distinction between information (message) and energy (signal). Wiener [28] established the distinction between signal and information, and associated "purposes" to goal states, or "consigns" in feedback loops. This way, the concepts described in cognitive language are anchored in Physics, Engineering and Mathematics. McCulloch [13] and von Neumann [26 constructed the modular automata theory and applied it to the formal description of neuronal networks and the synthesis of computers. Additionally, von Neumann addressed self-reproduction, self-reparation and tolerance to failure problems, which are nowadays also associated to agents (e.g. [2], [6], [7]).

With the arrival of AI, the initial formulations of connectionism (artificial neuronal networks and modular theory of deterministic and probabilistic automata) are partially obscured by new symbolic formulations based in rules. Here the inference is understood as a search process in a states space. Thus, the idea of "actors" appears, like a concurrent computation model in distributed systems [1. Along with object-oriented programming [15] these are the antecedents of 
the agents. Conceptually, it is Minsky [16] who raises the social idea of agency, essentially basing on "personification" of the verbs used in natural language to describe the necessary processes for the execution of a certain activity ("build", "see", "grasp", "move", "release"). The strategy to describe in natural language an agent's "beliefs", "desires" and "intentions" and later to develop the formal counterpart of the linguistic terms, is still used at the present time 23. In fact, a complete agent language is dominant in Software Engineering (SE) [19, and its importance also grows in Knowledge Engineering (KE) 3]. It is advisable to indicate that, as with the term of AI, in the agents field there is usually an abuse of excessively loaded cognitive nomenclature of anthropomorphous semantics. Finally, during implementation, there is no remedy than to reduce to algorithms and automata, and to entities and relations of the existing programming languages.

\section{Levels and Domains in Agent Models}

Since the introduction of the knowledge level by Newell [17, and Marr [10] called "theory of calculus" -, it is usual to describe the knowledge necessary to understand any calculation in three levels: physical $(P L)$, symbols $(S L)$ and knowledge $(K L)$. Or, in a simpler way: machine hardware, programs and models, and algorithms. Starting from the idea of reference systems in Physics and the proposals by Maturana [12] and Varela [25] in Biology, in 1978 [14] the figure of the external observer in computation is introduced. This gives rise to two description domains of the organizations and relations at each level: (1) the own domain of the level $(O D)$, where the causality is intrinsic and the semantics comes imposed by the structure and dynamics of the level, and, (2) the external observer domain $(E O D)$ to the computation carried out in that level, where the semantics is arbitrary and the interpretation of the calculation depends of the observer and, in general, of the application domain.

When superposing both domains $(O D, E O D)$, at the three levels $((K L, S L$, $P L$ ), we obtain a building of three plants and six apartments (two by plant), in which the agents reside (see Fig. (1). Thus, we have agents $(O D-P L),(E O D-P L)$, $(E O D-S L)$, etc. That is to say, the knowledge necessary to specify an agent may be decomposed into six elements: models $(E O D, O D)$, programs $(E O D, O D)$ and machines $E O D, O D$ ). When one or more of these components is the relevant one (assuming the existence of the others), this one gives the generic name to the agent. Thus, for example, robots are essentially physical agents because it is assumed that the greater complexity is associated to the design of its sensors and effectors, independently of their navigation programs. In an analogous manner, a great part of agents usual in IS is symbolic because it is assumed that most of its complexity is associated to the construction of a program whose interface is human (through screen and keyboard) and we do not need to worry about the design of the body of this type of agents.

Finally, the majority of the current most ambitious proposals are conceptual agents, at level of knowledge, and, more exactly, knowledge agents in the external 


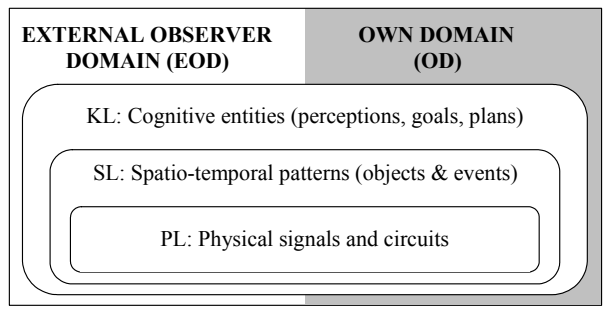

Fig. 1. The three nested levels $(P L, S L, K L)$ and the two domains $(E O D, O D)$ of description of the calculus performed by an agent

observer domain $(K L-E O D)$ because their implementation only exists in natural language. Those components of a $K L-E O D$ agent that may be formalized in terms of algorithms, ANN or FSA (that is to say, of its underlying formal model), already are $K L-O D$ agents, leaving all the own semantics of the EOD. For example, the intentions and desires are now sets (classes) and the transition diagrams among states (elements of a class) are now matrixes. Analogously, the beliefs (the knowledge base), when passing from $E O D$ to $O D$, are reduced to inferential rules, frames, logical entities, graphs or causal networks. And, most of the current architectures of "social agents" and "intentional agents" (BDI [22], for instance), are essentially developments at knowledge level and in the external observer domain.

\section{Computational Agent Conceptual Model (KL-EOD)}

Like in AI and Robotics, in the agency three basic architectures are distinguished - symbolic, situated and connectionist -, to approach different solutions to a problem, by modeling data and knowledge and later operating the inferences of the model. An agent is symbolic when it uses declaratory and explicit knowledge in natural language to describe the constituent organizations ("concepts") and the inference rules. In Robotics this is associated to "deliberative architectures", which spend time and a high number of computational resources in the decision process. The associated tasks usually are diagnosis, planning, and inductive and case-based reasoning (CBR) learning. Most knowledge-based systems (KBS) follow this paradigm. An agent is reactive or situated when knowledge representation is within two configuration tables of precalculated input and output, usually called "perceptions" and "actions". Here, the inference procedure is of "reflex" type (stimulus-response), very fast and adapted for real-time applications. The perception-action link is also given by a table or a automata with few states. It is proper for monitoring tasks and for the execution phase of motor planning, where a command is decomposed into a set of precalculated elementary actions that execute in "efficient" time, without having to deliberate. An agent is connectionist when knowledge representation is given in terms of labeled numerical 
lines, as much for the inputs as for the outputs, and the inference functions are adjustable numerical associators.

It is difficult to have all the necessary knowledge for an application. For that reason, the most frequent situations demand hybrid solutions, with reactive and deliberative, and with symbolic and connectionist parts. For that reason, in the agency paradigm, there are also hybrid architectures that combine agents of reactive and deliberative type. The reactive part reacts to the events of the environment without investing reasoning, whereas the deliberative support plans (it distributes the simplest goals) and performs tasks of superior abstraction level. Hybrid architectures are organized horizontally, so that the layers have access to sensors and actuators, and vertically, where a layer acts as an interface to sensors and actuators.

Finally, it is our conjecture that only two basic types of agent exist: (1) the ones based on descriptions in $E O D$ that use declarative knowledge in natural language, and, (2) those based on mechanisms of the $O D$, causal in the implementation of the three mentioned levels. Whichever the final version of the agent at knowledge level $(K L-E O D)$ should be, the following phase is to operationalize its entities and relations, its data and inferences. The formal model most used for the description of abstract agent architectures is automata theory.

\section{A Case Study in Visual Surveillance}

The previous concepts are being applied in semi-automatic visual surveillance tasks [5], [8, $, 9,[11,21,24]$ composed of a set of collaborative cameras installed in a building and a camera-mounted mobile robot to offer pre-alarms and/or alarms detected indoor and outdoor. The video images captured by each of the cameras enable segmenting and tracking objects of interest (obtained as image blobs) with the objective of providing meaningful events and suspicious activities. The cameras collaborate in the sense of obtaining richer surveillance observations that are only available through the fusion of information captured on various places. The mobile robot may be used if necessary to navigate to zones not monitored by fixed cameras or to dangerous places. People roaming or abandoning an object are some typical suspicious surveillance situations. After explaining this initial specification, an Analysis Overview Diagram 20] as shown in Fig. 2 is gotten.

The original diagram has been adapted to clearly show the three levels $(P L$, $S L$ and $K L)$ exposed previously. In this scheme there are physical agents $C a m-$ era, Robot and Alarm Center at PL. On the next level, $S L$, software agents (Camera Agent, Situation Agent, Social Agent, Coordinator Agent and Historic Agent), message events (Inform Event, Inform Situation, Command Camera Agent, Command Situation Agent and Alert Alarm Central), as well as believes data Image Database, Situation Database are shown. Lastly, the $K L$ includes goals, perceptions and actions, as described earlier. At top level, the main goal is Detect anomalous situation from which all subgoals are derived. Perceptions are also simplified into one general percept called Behavior annotated video. 


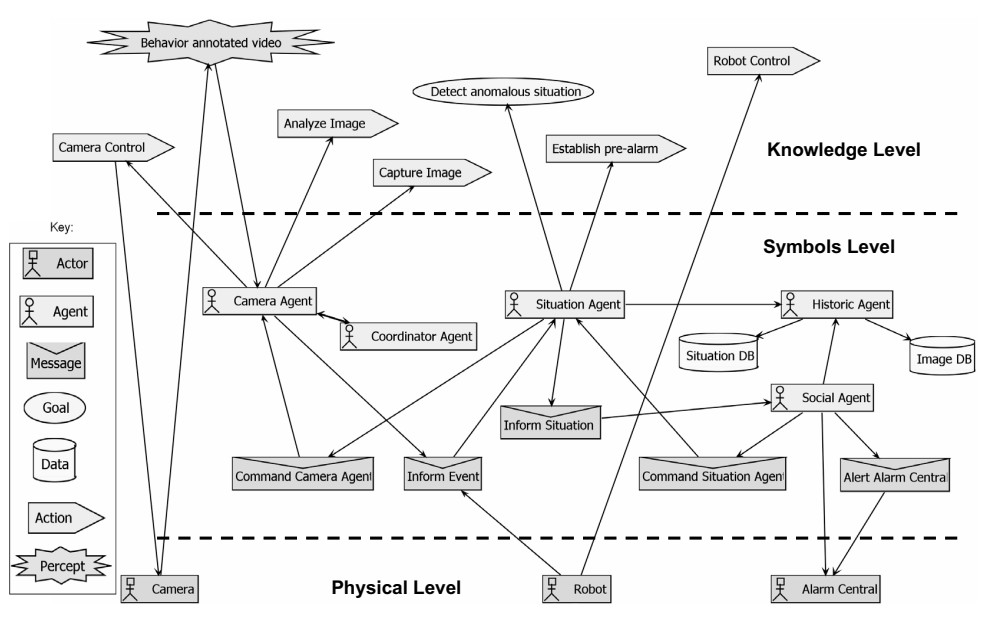

Fig. 2. Analysis Overview Diagram for a semi-automatic video-surveillance system

The actions shown in the figure are proper of any surveillance task faced from computer vision and robotics.

A more precise system specification is detailed next. A physical Camera provides the system (concretely to Camera Agent and Mobile Robot Agent) with the environment information captured. The Camera Agent has the ability to control the camera to move it in horizontal and vertical direction, and even change the zoom. This control is represented by means of action Camera Control. Similarly, Mobile Robot Agent has the ability to control the movements of the physical Robot (for example to advance, to turn down, to turn right or left, to accelerate, to stop, etc.). This ability is determined by means of action Robot Control. The Camera Agent analyzes input videos (it is represented as an action) to later send it to Historic Agent, which will store it in the Image Database. For example, an annotated image could be the face of a suspicious person. The Camera Agent is also in charge of studying an image or a sequence of images to understand what is happening in the field of vision. By means of this study, behaviors, actions, trajectories, etc., are obtained. The Mobile Robot Agent also incorporates these abilities (represented by actions Analyze Image and Capture Image) because the robot is also equipped with a built-in camera. The Camera Agent communicates the Situation Agent the events that it has detected. Now, Situation Agent has the objective to detect anomalous situations. In addition, it sends commands to the Mobile Robot Agent or Camera Agent (through message Command Camera Agent). It sends a command to the Camera Agent to communicate a need of coordination (when an event happens through several cameras), to request that it captures an image, or that it performs a zoom, etc. It sends a command to the mobile robot to navigate to the place where a possible suspicious event, which has fallen out of the visual field of the cameras, is taking place. The Situation Agent communicates with Historic Agent to ask for the events stored in the Situation Database. In this data base the situations and the behaviors considered 


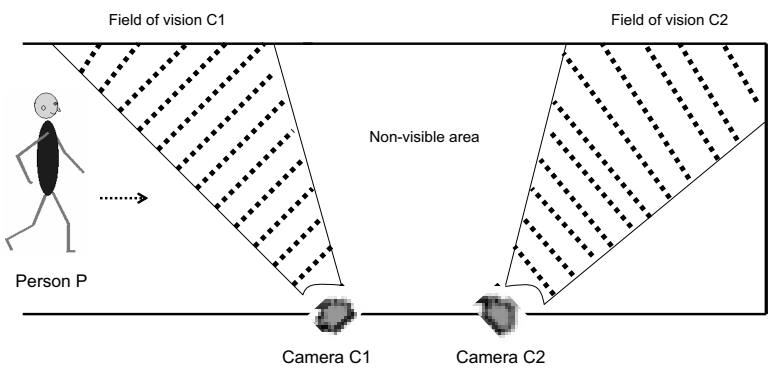

Fig. 3. A sidewalk with two non-overlapping cameras

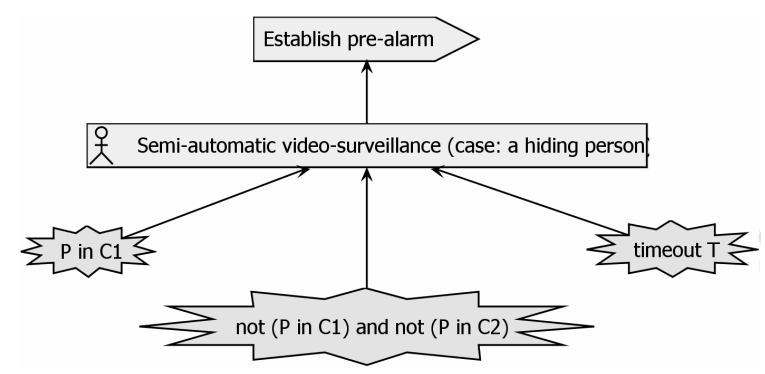

Fig. 4. Goal "Detect hiding person"

suspicious are stored. The Situation Agent communicates to Social Agent that a suspicious situation has happened (message Inform Situation). In the Situation Database the detected suspicious situations are registered.

Let us now approach one possible suspicious situation detection at $K L$ and represented in Fig 3. Two cameras are focusing on a sidewalk and a person is walking along it. One sub-goal of "Detect anomalous situation" is "Detect hiding person". As shown in Fig. 4, action Establish pre-alarm comes from consecutively analyzing perceptions "P in C1", "not (P in $\mathrm{C} 1$ ) and not $(\mathrm{P}$ in $\mathrm{C} 2)$ " and "timeout $\mathrm{T}$ " at knowledge level and in the external observer domain. The meanings for these perceptions are "the person $\mathrm{P}$ is present in the field of vision of camera 1 ", "the person $\mathrm{P}$ is neither present in the field of vision of camera 1, nor in the field

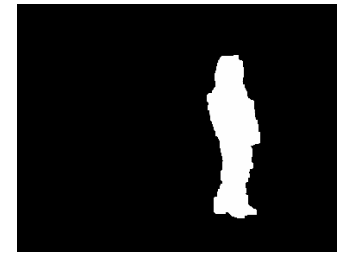

(a)

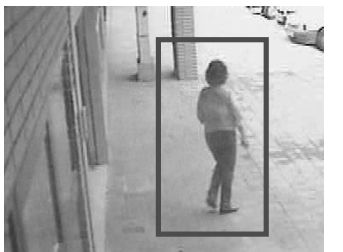

(b)

Fig. 5. Relation between $O D$ and $E O D$ in image segmentation. (a) Blob in the $O D$. (b) Person in the EOD. 
Table 1. $O D-E O D$ relation for goal "Detect hiding person" (simplified to a single camera)

\begin{tabular}{|c|c|}
\hline$\overline{\mathrm{OD}}$ & EOD \\
\hline $\begin{array}{l}\text { No blob at } t-1 \\
\text { No blob at } t\end{array}$ & $\begin{array}{l}\text { No person in camera } 1 \text { field of vision } \\
\text { [ ] }\end{array}$ \\
\hline $\begin{array}{l}\text { No blob at } t-1 \\
\text { Blob at the left part of image at } t\end{array}$ & $\begin{array}{l}\text { Person } \mathrm{P} \text { arrives to camera C1 field of vision from the } \\
\text { left } \\
{[\text { Arrives }(\mathrm{P}, \mathrm{L}, \mathrm{C} 1)]}\end{array}$ \\
\hline $\begin{array}{l}\text { No blob at } t-1 \\
\text { Blob at the right part of image at } t\end{array}$ & $\begin{array}{l}\text { Person } \mathrm{P} \text { arrives to camera C1 field of vision from the } \\
\text { right } \\
{[\text { Arrives }(\mathrm{P}, \mathrm{R}, \mathrm{C} 1)]}\end{array}$ \\
\hline $\begin{array}{l}\text { Blob at the left part of image at } t-1 \\
\text { Blob at any central part of image at } t\end{array}$ & $\begin{array}{l}\text { Person } \mathrm{P} \text { walks in camera } \mathrm{C} 1 \text { field of vision } \\
{[\text { Walks }(\mathrm{P}, \mathrm{C} 1)]}\end{array}$ \\
\hline $\begin{array}{l}\text { Blob at any central part of image at } t-1 \\
\text { Blob at any central part of image at } t\end{array}$ & $\begin{array}{l}\text { Person P walks in camera C1 field of vision } \\
{[\text { Walks }(\mathrm{P}, \mathrm{C} 1)]}\end{array}$ \\
\hline $\begin{array}{l}\text { Blob at the right part of image at } t-1 \\
\text { Blob at any central part of image at } t\end{array}$ & $\begin{array}{l}\text { Person P walks in camera C1 field of vision } \\
{[\text { Walks }(\mathrm{P}, \mathrm{C} 1)]}\end{array}$ \\
\hline $\begin{array}{l}\text { Blob at any central part of image at } t-1 \\
\text { Blob at the right part of image at } t\end{array}$ & $\begin{array}{l}\text { Person P leaves camera } \mathrm{C} 1 \text { field of vision to the right } \\
{[\text { Leaves }(\mathrm{P}, \mathrm{R}, \mathrm{C} 1)]}\end{array}$ \\
\hline $\begin{array}{l}\text { Blob at any central part of image at } t-1 \\
\text { Blob at the left part of image at } t\end{array}$ & $\begin{array}{l}\text { Person P leaves camera C1 field of vision to the left } \\
{[\text { Leaves }(\mathrm{P}, \mathrm{L}, \mathrm{C} 1)]}\end{array}$ \\
\hline
\end{tabular}

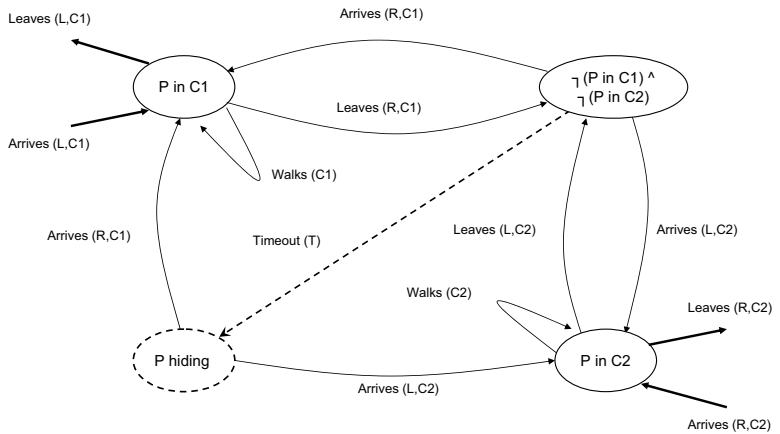

Fig. 6. Automata including state suspicious activity "Person hiding"

of vision of camera 2", and "a period superior to T has elapsed since the person $\mathrm{P}$ left the field of vision of camera 1 in the direction of camera 2" respectively. An "intelligent" system should consider this as a suspicious situation. Evidently, this representation may only be described at $E O D$, where the information has passed from the $O D$ through a domain dependent knowledge injection.

Fig. 5 shows a simple example of the relation between $O D$ and EOD in a typical visual surveillance task. In the $O D$, there is a segmented blob with parameters height, length, ..., obtained at instant $t$. (b) In the external observer domain, there is a person P "walking", tracked by camera C. In this sense, Table 1 shows both domains for goal "Detect hiding person".

Also, as explained in this paper, the operationalization of the computational agent conceptual model $(K L-E O D)$ may be formally described as finite state automata. Fig. 6] shows the four states necessary to model all possibilities for a person walking through the sidewalk described in Fig. 3. The automaton covers 


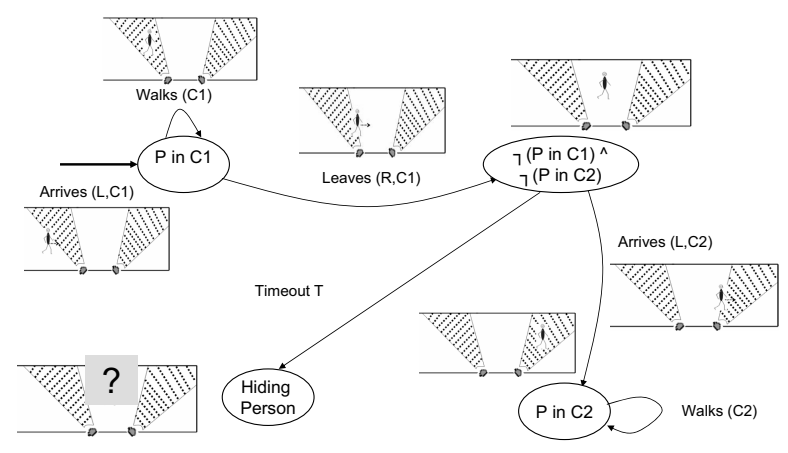

Fig. 7. Sub-automata illustrating the detection of activity "Person hiding"

all the EOD semantic richness previously explained. Also Fig. 7 is offered illustrating one possible path towards detecting a hiding person in the scenario described.

\section{Conclusions}

In this paper the agent concept has been faced from a computational perspective. Therefore, it has been shown that a computational agent must specify its conceptual model, its formal model and its implementation, starting from the set of functional specifications available on its goals, activities and tasks. We are currently engaged in modeling the visual surveillance task. Surveillance systems consist of a great diversity of entities that have to cooperate in highly dynamic and distributed environments. The use of agents for their control allows a greater degree of autonomy and response because of their capabilities to adapt and to cooperate.

\section{Acknowledgements}

This work was partially supported by Spanish Ministerio de Ciencia e Innovación TIN2007-67586-C02-02 grant, and by Junta de Comunidades de CastillaLa Mancha PII2I09-0069-0994, PII2I09-0071-3947 and PEII09-0054-9581 grants.

\section{References}

1. Agha, G.: Actors: A Model of Concurrent Computing in Distributed Systems. The MIT Press, Cambridge (1986)

2. Bertier, M., Marin, O., Sens, P.: Performance analysis of a hierarchical failure detector. In: Proceedings of the International Conference on Dependable Systems and Networks, DSN 2003, pp. 635-644 (2003) 
3. Cuena, J., Demazeau, Y., Garcia-Serrano, A., Treur, J.: Knowledge Engineering and Agent Technology. Frontiers in Artificial Intelligence and Applications, vol. 52. IOS Press, Amsterdam (2003)

4. Franklin, S., Graesser, A.: Is it an agent, or just a program?: A taxonomy for autonomous agents. In: Jennings, N.R., Wooldridge, M.J., Müller, J.P. (eds.) ECAIWS 1996 and ATAL 1996. LNCS, vol. 1193, pp. 121-135. Springer, Heidelberg (1997)

5. Gascueña, J.M., Fernández-Caballero, A.: The INGENIAS methodology for advanced surveillance systems modelling. In: Mira, J., Álvarez, J.R. (eds.) IWINAC 2007. LNCS, vol. 4528, pp. 541-550. Springer, Heidelberg (2007)

6. Guessoum, Z., Briot, J.P., Marin, O., Hamel, A., Sens, P.: Dynamic and adaptive replication for large-scale reliable multi-agent systems. In: Proceedings of the 1st International Workshop on Software Engineering for Large-Scale Multi-Agent Systems, SELMAS 2002, pp. 26-30 (2002)

7. Jiang, Y., Xia, Z., Zhong, Y., Zhang, S.: The construction and analysis of agent fault-tolerance model based on pi-calculus. In: Bubak, M., van Albada, G.D., Sloot, P.M.A., Dongarra, J. (eds.) ICCS 2004. LNCS, vol. 3038, pp. 591-598. Springer, Heidelberg (2004)

8. López, M.T., Fernández-Caballero, A., Fernández, M.A., Mira, J., Delgado, A.E.: Dynamic visual attention model in image sequences. Image and Vision Computing 25(5), 597-613 (2007)

9. López, M.T., Fernández-Caballero, A., Fernández, M.A., Mira, J., Delgado, A.E.: Visual surveillance by dynamic visual attention method. Pattern Recognition 39(11), 2194-2211 (2006)

10. Marr, D.: Vision. Freeman, San Francisco (1982)

11. Martínez, R., Rincón, M., Bachiller, M., Mira, J.: On the correspondence between objects and events for the diagnosis of situations in visual surveillance tasks. Pattern Recognition Letters 29(8), 1117-1135 (2008)

12. Maturana, H.R.: The organization of the living: A theory of the living organization. International Journal of Man-Machine Studies 7, 313-332 (1975)

13. McCulloch, W.S.: Embodiments of Mind. The MIT Press, Cambridge (1965)

14. Mira, J., Delgado, A.E., Moreno-Diaz, R.: Cooperative processes in cerebral dynamic. Applications of Information and Control Systems 3, 273-280 (1979)

15. Meyer, B.: Object-Oriented Software Construction. Prentice Hall, Englewood Cliffs (1997)

16. Minsky, M.L.: Steps towards Artificial Intelligence. Proceedings of the Institute of Radio Engineers 49, 8-30 (1961)

17. Newell, A., Simon, H.A.: Human Problem Solving. Prentice Hall, Englewood Cliffs (1972)

18. Nunes de Castro, L.: Fundamentals of Natural Computing: Basic Concepts, Algorithms, and Applications. Chapman \& Hall/CRC, Boca Raton (2006)

19. Padgham, L., Zambonelli, F.: AOSE VII / AOSE 2006. LNCS, vol. 4405. Springer, Heidelberg (2007)

20. Padgham, L., Winikoff, M.: Developing Intelligent Agents Systems: A Practical Guide. John Wiley and Sons, Chichester (2004)

21. Pavón, J., Gómez-Sanz, J., Fernández-Caballero, A., Valencia-Jiménez, J.J.: Development of intelligent multi-sensor surveillance systems with agents. Robotics and Autonomous Systems 55(12), 892-903 (2007)

22. Rao, A.S., Georgeff, M.P.: Modeling rational agents within a BDI-architecture. In: Proceedings of the 2nd International Conference on Principles of Knowledge Representation and Reasoning, KR 1991, pp. 473-484 (1991) 
23. Russell, S., Norvig, P.: Artificial Intelligence: A Modern Approach, 2nd edn. Prentice Hall, Englewood Cliffs (2002)

24. Valencia-Jiménez, J.J., Fernández-Caballero, A.: Holonic multi-agent systems to integrate independent multi-sensor platforms in complex surveillance. In: Proceedings of the IEEE International Conference on Advanced Video and Signal based Surveillance, AVSS 2006, p. 49 (2006)

25. Varela, F.J.: Principles of Biological Autonomy. North-Holland, Amsterdam (1979)

26. von Neumann, J.: The Computer and the Brain. Yale University Press, New Haven (1958)

27. Weiss, G.: Multiagent Systems: A Modern Approach to Distributed Artificial Intelligence. The MIT Press, Cambridge (1999)

28. Wiener, N.: Cybernetics: Or the Control and Communication in the Animal and the Machine. Wiley, Chichester (1961) 